Check for updates

Cite this: RSC Adv., 2018, 8, 18771

Received 16th January 2018

Accepted 23rd April 2018

DOI: $10.1039 / c 8 r a 00464 a$

rsc.li/rsc-advances

\section{Supramolecular hydrogels encapsulating bioengineered mesenchymal stem cells for ischemic therapy $\dagger$}

\author{
Byung Woo Hwang, ${ }^{a}$ Young-Eun Kim, (ID b Mungu Kim, ${ }^{\mathrm{c}}$ Seulgi Han, ${ }^{\mathrm{b}}$ Seoyeon Bok, \\ Kyeng Min Park, ${ }^{d}$ Annadka Shrinidhi, ${ }^{d}$ Ki Su Kim, ${ }^{\text {ce }}$ G-One Ahn ${ }^{\text {b }}$ \\ and Sei Kwang Hahn (iD *ac
}

We developed supramolecular hyaluronate (HA) hydrogels to encapsulate genetically engineered mesenchymal stem cells (MSCs) for the treatment of limb ischemia. In vivo angiogenic factors could be produced stably by the bioengineered MSCs (BMSCs) within the supramolecular hydrogels showing effective vascular repair and enhanced blood perfusion.
Clinical limb ischemia (CLI) is the severe manifestation of peripheral arterial disease, which is one of the most common diseases in the population over 70 years old, up to $20 \% .^{1}$ Especially for $30 \%$ of the patients who suffer from CLI, amputation is the only remaining method, because the conventional treatments such as medication, endovascular therapy and arterial surgery are not very helpful. ${ }^{2}$ Revascularization is necessary to restore the damaged tissue without amputation. Revascularization is a sequential and sophisticated process related to various signaling molecules participating in matrix reconstruction, migration and assembly. ${ }^{3}$ To accelerate the revascularization in ischemic areas, therapeutic treatment aims to deliver concentrated angiogenic factors locally, such as vascular endothelial growth factor A (VEGF-A) and hepatocyte growth factor (HGF). VEGF-A is a well-known angiogenic factor, participating in the early stage of vessel sprouting. HGF is also one of the representative angiogenic factors. HGF is involved in mitogenesis, migration of epithelial cells, as well as their 3dimensional organization and generation of new blood capillaries. ${ }^{4}$ Neovascularization is known to be synergistically promoted by the combined delivery of HGF and VEGF-A, because their signaling pathways are co-regulated during cellular migration and endothelial morphogenesis. ${ }^{5}$

${ }^{a}$ Department of Materials Science and Engineering, Pohang University of Science and Technology (POSTECH), 77 Cheongam-ro, Pohang 37673, Korea

${ }^{b}$ Division of Integrative Biosciences and Biotechnology, Pohang University of Science and Technology (POSTECH), 77 Cheongam-ro, Pohang 37673, Korea

${ }^{c}$ PHI BIOMED Co., 175 Yeoksam-ro, Gangnam-gu, Seoul 06247, Korea

${ }^{d}$ Center for Self-assembly and Complexity (CSC), Institute for Basic Science (IBS), Pohang 37673, Republic of Korea

${ }^{e}$ Department of Organic Materials Science and Engineering, College of Engineering, Pusan National University, 2 Busandaehak-ro 63 beon-gil, Gumjeong-gu, Busan, 46241, Korea

$\dagger$ Electronic supplementary information (ESI) available. See DOI: 10.1039/c8ra00464a
With the progress of tissue engineering technology, therapeutic neovascularization has been investigated with 3 different strategies for the efficient angiogenic factor delivery. ${ }^{6}$ First, angiogenic factors have been directly delivered to contribute to the formation of blood vessels. However, there have been few clinical trials for the direct protein delivery, since their activities cannot be maintained for a sufficient time to regenerate blood vessels. ${ }^{6}$ Second, gene therapy has been investigated for the manipulation of host chromosomes by gene delivery with viral or non-viral vectors including adeno-associated virus, lenti-virus and synthetic polymer vesicles. ${ }^{7}$ This can raise the angiogenic protein concentration efficiently, but the relevant clinical applications have been limited for limb ischemic diseases. ${ }^{8}$ As is well known, there is a considerable risk of an adverse immune response and the overexpression of certain angiogenic factors causing a carcinogenic environment. ${ }^{7,9}$

Lastly, cell therapy has been performed using stem/ progenitor cells which can differentiate into vasculature or secrete angiogenic factors. ${ }^{\mathbf{1 0}}$ Cell therapy has attracted a great attention defining individual stem/progenitor cells involved in the revascularization processes. ${ }^{11}$ Although a few examples with substantial improvement have been reported for the clinical trial of cell therapy, ${ }^{12}$ rapid clearance and low viability are significant challenges for the success of cell therapy.

Recently, therapeutic cells are genetically modified for the controlled delivery of angiogenic factors to overcome both the low efficiency of direct protein delivery and the risk of viral-mediated gene delivery. However, rapid clearance and low survival rate of therapeutic cells are still technical hurdles for further development. Diverse studies have been actively conducted to develop various scaffolds to increase the cell survival rate and prevent the rapid clearance of therapeutic cells. ${ }^{13}$ As an artificial extracellular matrix 
(ECM), hydrogels have been developed to support cells providing a biocompatible environment similar with living tissues in the body.

In this work, we hypothesized that the co-delivery of VEGF-A and HGF released by bioengineered mesenchymal stem cells (BMSCs) might be one of the best approaches for neovascularization in ischemic diseases. BMSCs might maintain high expression levels of HGF and VEGF-A within the artificial ECM of hydrogels. A variety of supramolecular hydrogels have been developed using a strong host-guest combination of cucurbituril. ${ }^{\mathbf{1 4 , 1 5}}$ We previously designed a novel system for long-term and safe delivery of therapeutic proteins by the BMSCs encapsulated in supramolecular hyaluronate (HA) hydrogels. ${ }^{16,17}$ Supramolecular HA hydrogels can localize and protect genetically modified MSCs from the host immune systems. ${ }^{18}$ In addition, supramolecular hydrogels can be injected without toxic chemical reaction due to the fast crosslinking by the strong host-guest interaction between cucurbit[6]uril (CB [6]) and diaminohexane (DAH) attached to the HA backbone. ${ }^{15}$ We assessed the therapeutic efficacy of BMSCs releasing both HGF and VEGF-A in supramolecular hydrogels by laser Doppler imaging (LDI).

To achieve the high crosslinking density and facile synthesis, we carried out mono-functionalization of $\mathrm{CB}[6]$, which is a big challenge for various biomedical applications ${ }^{19,20}$ (Fig. 1A). Despite the great effort to introduce functional moieties on $\mathrm{CB}$ $[6],{ }^{21}$ mono-amination of $\mathrm{CB}[6]$ is still difficult and complicated. ${ }^{22,23}$ We previously reported the method to synthesize CB [6]-HA derivative via Michael addition between thiol ( $\mathrm{SH}$ ) modified HA and mono-allyloxy $\mathrm{CB}[6]$. However, there were unreacted thiol groups on HA backbone, resulting in selfcrosslinking by disulfide bonding. ${ }^{24,25}$ Thus, direct functionalization of $\mathrm{CB}[6]$ was needed to avoid side-reaction by the reactive $\mathrm{SH}$ group.

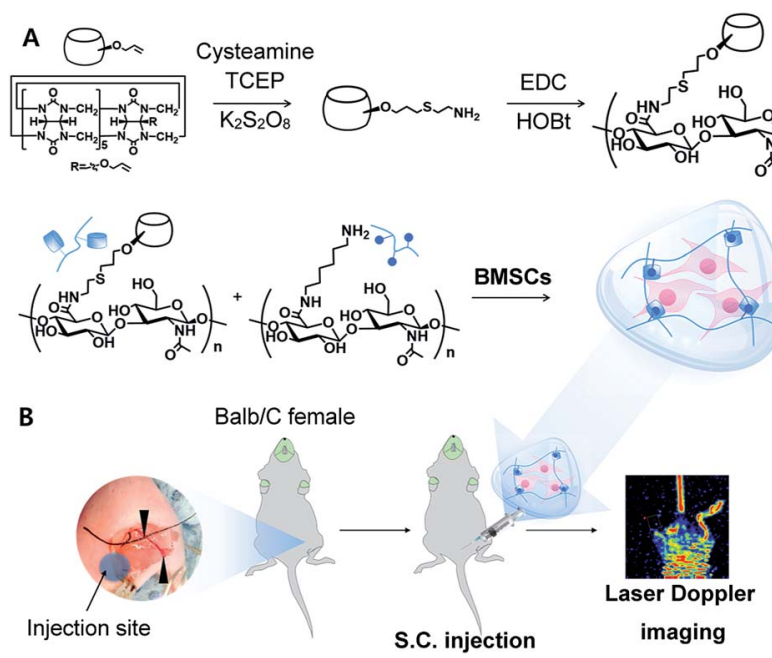

Fig. 1 Schematic illustration for (A) sequential synthesis of $m C B[6]-H A$ using mono-amine $\mathrm{CB}[6]$ and the preparation of $\mathrm{mCB}[6] / \mathrm{DAH}$ hydrogels, and (B) in vivo tests using BMSCs with the analysis by LDI to investigate ischemic limb reperfusion. Black arrows indicate the ligation point for hind limb surgery.
First, mono-allyloxy $\mathrm{CB}[6]$ was synthesized as previously reported elsewhere. ${ }^{\mathbf{2 4 2 5}}$ To introduce amine groups to monoallyloxy $\mathrm{CB}[6]$, cysteamine was conjugated in the presence of reducing tris(2-carboxyethyl)phosphine (TCEP) and potassium persulfate $\left(\mathrm{K}_{2} \mathrm{~S}_{2} \mathrm{O}_{8}\right)$ as an initiator. The product, monoaminated $\mathrm{CB}[6](\mathrm{mCB}[6])$ was purified by washing with methanol and acetone sequentially.

In this reaction, TCEP was essential to prevent the formation of the inclusion between $\mathrm{mCB}[6]$ and cysteamine. Since cystamine, a disulfide form of cysteamine, interacts more strongly with the guest-molecule of $\mathrm{CB}[6]$ than $\mathrm{DAH}, \mathrm{CB}[6]$ cannot make the host-guest interaction with DAH for the inclusion formation. After reaction at $70{ }^{\circ} \mathrm{C}$ for $24 \mathrm{~h}$, we could confirm mCB[6] was successfully synthesized and the inclusion of cystamine in $\mathrm{mCB}[6]$ was inhibited in the presence of TCEP (Fig. $2 \mathrm{~A}$ and $\mathrm{S} 1 \dagger$ ). The ${ }^{1} \mathrm{H}$ NMR spectrum showed the characteristic peaks of amine-arms $\left(-\mathrm{OCH}_{2} \mathrm{CH}_{2} \mathrm{CH}_{2}-\mathrm{S}-\mathrm{CH}_{2} \mathrm{CH}_{2}-\mathrm{NH}_{2}\right)$ at $\delta 3.1,2.77$, 2.63, 2.22 (Fig. 2A).

Despite the presence of mono-, di-amine and trifunctionalized $\mathrm{CB}[6]$ detected in MALDI-TOF MS spectroscopy (Fig. S1 $\dagger$ ), the majority of the compounds was found to be mono-amine functionalized $\mathrm{CB}[6]$, as shown by ${ }^{1} \mathrm{H}$ NMR (Fig. 2A). Then, we synthesized $\mathrm{mCB}[6]-\mathrm{HA}$ conjugate by using
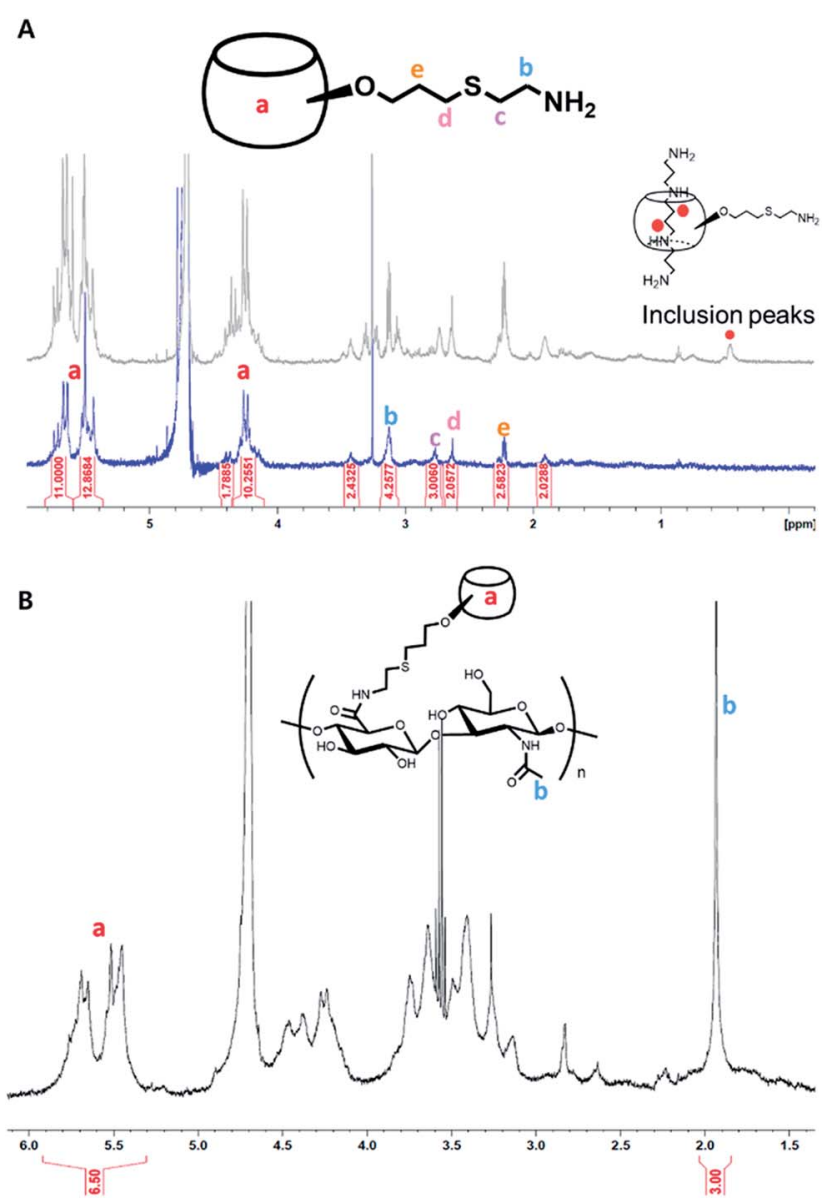

Fig. $2{ }^{1} \mathrm{H}$ NMR analysis for $(A) m C B[6]$, the formation of its inclusion with spermine, and (B) $\mathrm{mCB}[6]-\mathrm{HA}$. 
the simple EDC chemistry. There was no unreacted group in the final product. Moreover, the substitution rate was incredibly enhanced up to $28 \%$ (Fig. 2B). DAH-HA conjugates, a counterpart to $\mathrm{mCB}[6]-\mathrm{HA}$, were synthesized and characterized as we described elsewhere. ${ }^{26}$ According to the MTT assay (Fig. S2 $\dagger$ ), the hydrogel precursors of $\mathrm{mCB}[6]-\mathrm{HA}$ and DAH-HA showed little cytotoxicity at the synthetic concentrations of the hydrogel below $3 \mathrm{wt} / \mathrm{v} \%$.

The simple mixing of mCB[6]-HA and DAH-HA resulted in the rapid formation of $\mathrm{mCB}[6] / \mathrm{DAH}-\mathrm{HA}$ hydrogels within $30 \mathrm{~s}$. We have synthesized hydrogels using $\mathrm{mCB}[6]-\mathrm{HA}$ with a substitution ratio of $7.1 \%$ and $21.5 \%$, and confirmed in vitro degradation with and without hyaluronidase for 2 weeks (Fig. S3†). As a result, the highly substituted hydrogel (21.5\%) degraded by $c a$. $40 \%$ for 2 weeks, whereas the low substituted hydrogel $(7.1 \%)$ was decomposed by $c a$. $70 \%$ in the presence of hyaluronidase. The both groups showed little degradation (less than 10\%) in the absence of hyaluronidase. In our previous study, ${ }^{24}$ because $7 \%$ substituted hydrogels degraded slowly in mice for up to 3 months, the $21.5 \%$ substituted $\mathrm{mCB}[6] / \mathrm{DAH}-\mathrm{HA}$ hydrogels will remain for more than 3 months.

We used human-derived umbilical cord MSCs, which were kindly provided from Genexine Co. after genetic modification to express angiogenic factors such as HGF and VEGF-A using adenoviral vectors (Fig. 3A). MSCs expressing VEGF-A (BMSCs/ VEGF-A) and/or HGF (BMSCs/HGF) were added to the mCB[6]HA precursor solutions, and then mixed with the DAH-HA precursor solution. The self-assembly of $\mathrm{CB}[6]$ and $\mathrm{DAH}$ resulted in the immediate hydrogel formation encapsulating BMSCs homogeneously (Fig. 1B). To confirm in vitro gene expression of BMSCs with and without the $\mathrm{mCB}[6] / \mathrm{DAH}-\mathrm{HA}$ hydrogels,

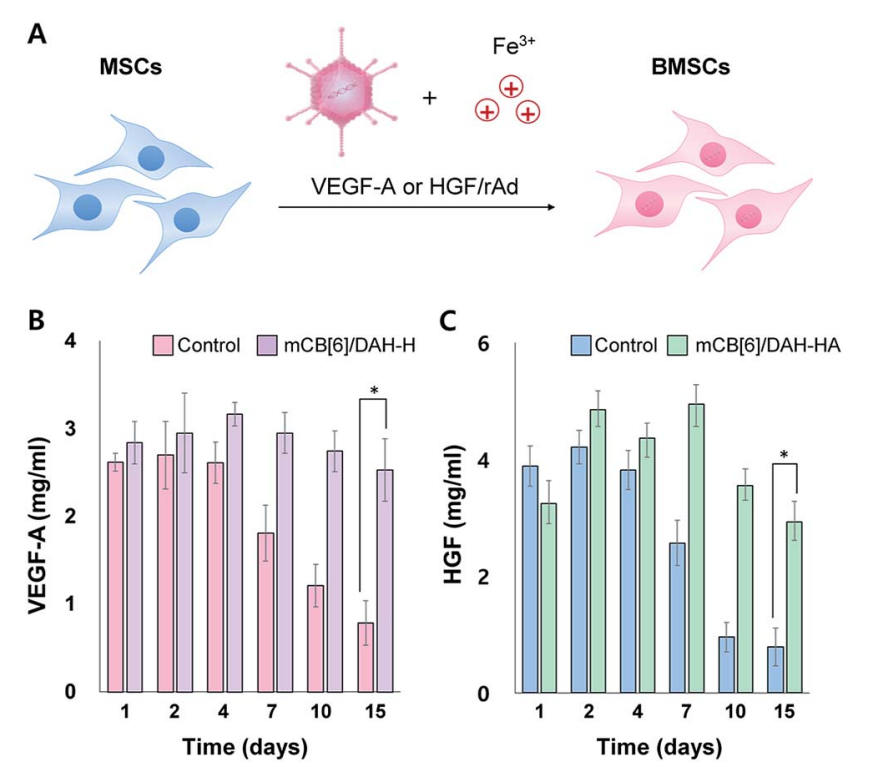

Fig. 3 (A) Genetic modification of MSCs using recombinant adenoviral $(\mathrm{rAd})$ vectors in the presence of $\mathrm{Fe}^{3+}$ ions to express VEGF-A and HGF. (B) VEGF-A expression levels and (C) HGF expression levels of BMSCs $\left(1 \times 10^{5}\right.$ cells per $\left.\mathrm{mL}\right)$ without and with the $\mathrm{mCB}[6] / \mathrm{DAH}-\mathrm{HA}$ hydrogels on PET membrane transwell inserts (mesh size $=8 \mu \mathrm{m}$ ) for specified periods over 2 weeks (mean $\pm \mathrm{SD}, n=3, * P<0.0001$ ).
BMSC/VEGF-A and BMSC/HGF were cultured in the hydrogel and the expression level of VEGF-A and HGF was analysed by ELISA for 15 days (Fig. 3B and C). As compared with the control group, the expression of VEGF-A and HGF was not so much different for the first and fourth days, but the difference became significant after a week. At day 15, while the expression level of the control group was reduced to less than one third, that of the hydrogel group was maintained at the same level with the initial level.

The animal model of hind limb ischemia was prepared by excision and ligation of superficial femoral artery using 6 to 8 week old BALB/c mice anesthetized with 5\% isoflurane (Fig. 1B). After $24 \mathrm{~h}$ post-surgery, we subcutaneously injected various samples of only medium (G1), $5 \times 10^{5}$ BMSCs/HGF in $100 \mu \mathrm{L}$ of medium (G2), the precursor solutions of matrixen (G3) and mCB[6]/DAH-HA hydrogels (G4). The blood perfusion of the ligated and the control limb was measured and compared by laser Doppler imaging (LDI; Perimed, North Royalton, Ohio) at the predetermined days (day $0,2,10$ and 24) post-therapy. The

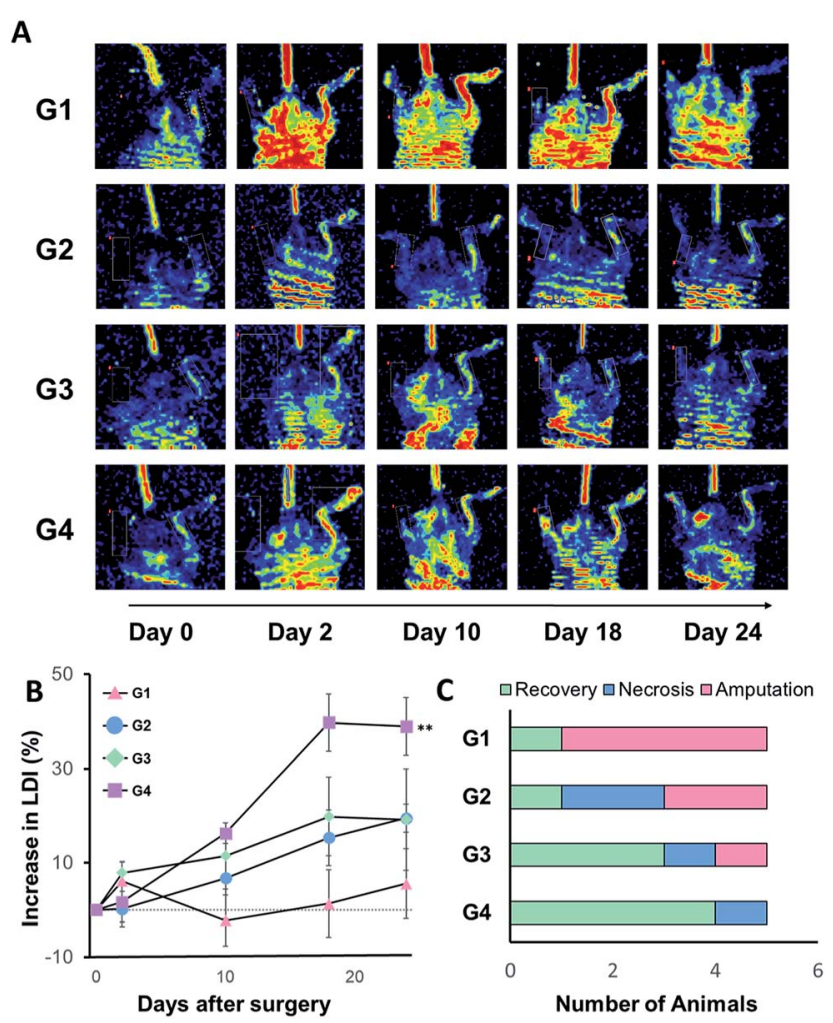

Fig. 4 Laser Doppler imaging (LDI) analysis for the blood reperfusion of BALB/c ischemic limb model mice after injection of BMSCs/HGF in various carriers. (A) Representative LDI images for peripheral blood flow in groups of mice that received media only (G1), BMSC/HGF in media (G2), in matrixen (G3), and in CB[6]/DAH-HA hydrogel (G4) at day $0,2,10,18$ and 24 . The color-coded image shows the blood flow distribution. The low or no perfusion is shown in blue and the highest perfusion is shown in red. (B) The quantitative analysis of blood flow in hind limbs expressed as the perfusion ratio of ischemic hind limb to the untreated opposite limb ( $n=5, * * P<0.01$ vs. the control of G1). (C) The number of animals categorized in 3 groups as fully recovered, necrotized and amputated limbs at day 24 post-injection. 
group treated with BMSCs/HGF encapsulated in $\mathrm{mCB}[6] / \mathrm{DAH}-$ HA hydrogels showed higher blood perfusion than other groups (Fig. 4A and B). Most of the limbs were amputated in the control group, but only one limb was necrotized in the group of eMSCs/ HGF within mCB[6]/DAH-HA hydrogels (Fig. 4C).

Based on these results, we assessed the combination effect of HGF and VEGF-A for ischemic limb reperfusion after subcutaneous injection of intact MSCs (G1), BMSCs/VEGF-A (G2), BMSCs/HGF (G3), and BMSCs/VEGF-A + HGF (G4) mixed with $100 \mu \mathrm{L}$ of the precursor solution of $\mathrm{mCB}[6] / \mathrm{DAH}-\mathrm{HA}$, respectively. The total population of cells in the hydrogels was $5 \times 10^{5}$ per heads. As shown in Fig. 5, VEGF-A and HGF produced by BMSCs helped the recovery of blood perfusion in the ischemic mice. The blood perfusion after treatment with BMSCs/VEGF-A or BMSCs/HGF was recovered more significantly than that of the intact MSCs. The best therapeutic effect was observed in the combination group of BMSCs/VEGF-A + HGF (Fig. 5A and B). After 28 days post-injection, all the limbs were fully recovered in every mouse except one mouse with gangrenous toe (Fig. 5C).
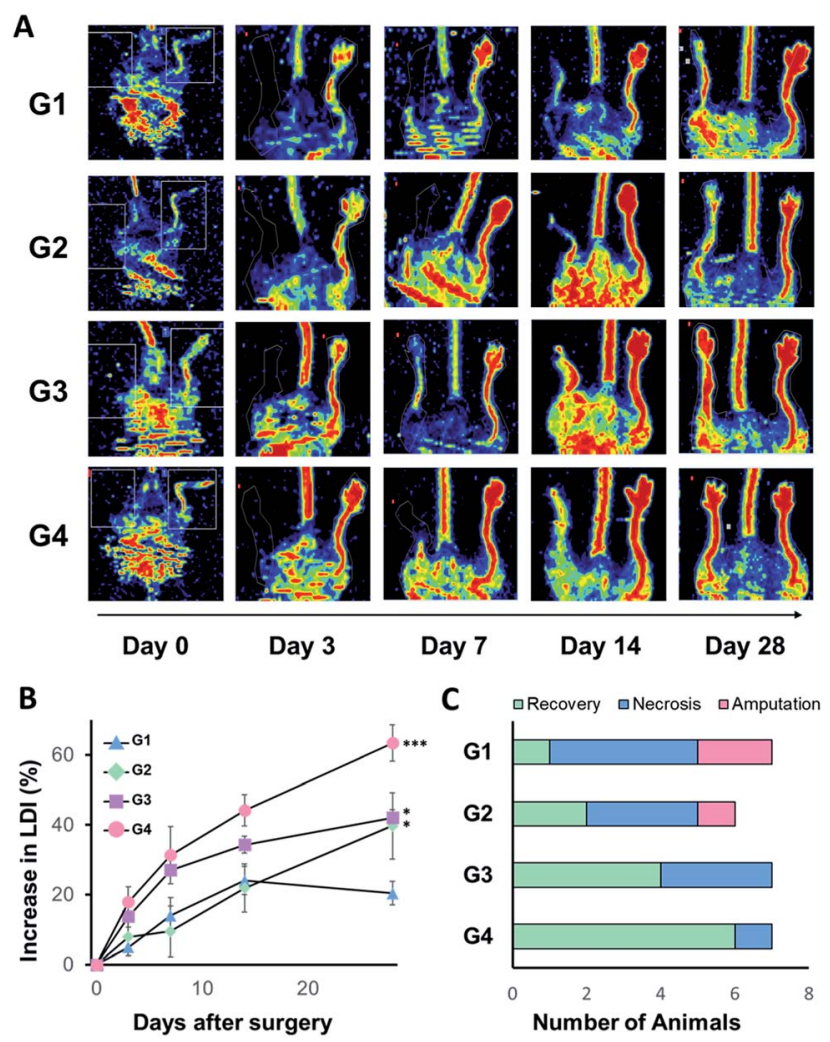

Fig. 5 Laser Doppler imaging (LDI) analysis for the blood reperfusion of BALB/c ischemic limb model mice after injection of various BMSCs in $\mathrm{mCB}[6] / \mathrm{DAH}-\mathrm{HA}$ hydrogels. (A) Representative LDI images for peripheral blood flow in groups of mice that received the intact MSC (G1), BMSC/VEGF-A (G2), BMSC/HGF (G3), and BMSC/HGF + VEGF-A (G4) within mCB[6]/DAH-HA hydrogels at day $0,3,7,14$ and 28 postinjection. The color-coded image shows blood flow distribution as in Fig. 4(B). (B) The quantitative analysis of blood flow in hind limbs expressed as the perfusion ratio of ischemic hind limb to the untreated opposite limb ( $n=6-7, * * * P<0.001$ vs. the control of G1). (C) The number of animals categorized in 3 groups as fully recovered, necrotized and amputated limbs at day 28 post-injection.
All animal experiments were performed in accordance with the National Institutes of Health Guide for the Care and Use of Experimental Animals and approved by the Institutional Animal Care and Use Committee of the Pohang University of Science and Technology.

The biological half-life of VEGF-A and HGF in the body is less than $50 \mathrm{~min}$ and $3 \mathrm{~min}$, respectively. ${ }^{27}$ Despite the short halflife, the massive administration of VEGF-A can cause the catastrophic blood vessel formation at the undesirable site. In addition, c-Met, the receptor of HGF, is implicated in the oncogenesis, which can be activated by the overexpressed $\mathrm{HGF}^{28}$ Thus, it is crucial to deliver an adequate level of angiogenic factors consistently for the angiogenic therapy. Our BMSCs/VEGF-A + HGF encapsulated in CB[6]/DAH-HA hydrogels might help to maintain the concentration of angiogenic factors continuously for the treatment of ischemia.

\section{Conclusions}

We developed a new ischemic therapy system to deliver angiogenic factors for long-term neovascularization using HGF and VEGF-A expressing BMSCs encapsulated in supramolecular mCB[6]/DAH-HA hydrogels. The BMSCs in the supramolecular HA hydrogels continuously expressed both VEGF-A and HGF, and released the angiogenic factors for a long-term period. The laser Doppler imaging clearly visualized the effective vascular repair and the enhanced blood perfusion in hind-limb ischemia model mice. Our angiogenic factor producing system using BMSCs in the $\mathrm{CB}[6] / \mathrm{DAH}-\mathrm{HA}$ hydrogels might be a promising candidate for various neovascularization therapies.

\section{Conflicts of interest}

There is no conflict to declare.

\section{Acknowledgements}

This research was supported by the grant of TIPS Project, Ministry of SMEs and Startups, Republic of Korea (S2557811). This research was also supported by the Basic Science Research Program (No. 2017R1E1A1A03070458) and the Bio \& Medical Technology Development Program (No. 2012M3A9C6049791) of the National Research Foundation (NRF) funded by the Ministry of Science and ICT, Korea.

\section{Notes and references}

1 A. Dua and C. J. Lee, Tech Vasc Intervent Radiol, 2016, 19, 9195.

2 N. W. Shammas, Vasc. Health Risk Manage., 2007, 3, 229-234.

$3 \mathrm{~J}$. Rouwkema and A. Khademhosseini, Trends Biotechnol., 2016, 34, 733-745.

4 F. Bussolino, M. F. Di Renzo, M. Ziche, E. Bocchietto, M. Olivero, L. Naldini, G. Gaudino, L. Tamagnone, A. Coffer, P. M. Comoglio, M. F. Di Renzo, M. Ziche, E. Bocchietto, M. Olivero, L. Naldini, G. Gaudino, L. Tamagnone, A. Coffer, P. M. Comoglio, M. F. Di Renzo, 
M. Ziche, E. Bocchietto, M. Olivero, L. Naldini, G. Gaudino, L. Tamagnone, A. Coffer and P. M. Comoglio, J. Cell Biol., 1992, 119, 629-641.

5 E. Sulpice, S. Ding, B. Muscatelli-Groux, M. Bergé, Z. C. Han, J. Plouet, G. Tobelem and T. Merkulova-Rainon, Biol. Cell, 2009, 101, 525-539.

6 H. Chu and Y. Wang, Ther. Delivery, 2012, 3, 693-714.

7 T. A. Khan, F. W. Sellke and R. J. Laham, Gene Ther., 2003, 10, 285-291.

8 R. Gupta, J. Tongers and D. W. Losordo, Circ. Res., 2009, 105, 724-736.

9 N. Horiguchi, H. Takayama, M. Toyoda, T. Otsuka, T. Fukusato, G. Merlino, H. Takagi and M. Mori, Oncogene, 2002, 21, 1791-1799.

10 D. P. Sieveking and M. K. Ng, Vasc. Med., 2009, 14, 153-166.

11 R. A. Brenes, M. Bear, C. Jadlowiec, M. Goodwin, P. Hashim, C. D. Protack, K. R. Ziegler, X. Li, L. S. Model, W. Lv, M. J. Collins and A. Dardik, Vascular, 2012, 20, 360-368.

12 H. Lawall, P. Bramlage and B. Amann, J Vasc Surg, 2011, 53, 445-453.

13 B. W. Hwang, S. J. Kim, K. M. Park, H. Kim, J. Yeom, J. Yang, H. Jeong, H. Jung, K. Kim, Y. C. Sung and S. K. Hahn, J. Controlled Release, 2015, 220, 119-129.

14 E. A. Appel, X. J. Loh, S. T. Jones, F. Biedermann, C. A. Dreiss and O. A. Scherman, J. Am. Chem. Soc., 2012, 134, 1176711773.

15 K. M. Park, J. Yang, H. Jung, J. Yeom, J. S. Park, K. Park, A. S. Hoffman, S. K. Hahn and K. Kim, ACS Nano, 2012, 6, 2960-2968.

16 K. Ozawa, K. Sato, I. Oh, K. Ozaki, R. Uchibori, Y. Obara, Y. Kikuchi, T. Ito, T. Okada, M. Urabe, H. Mizukami and A. Kume, J. Autoimmun., 2008, 30, 121-127.
17 K. C. Rustad, V. W. Wong, M. Sorkin, J. P. Glotzbach, M. R. Major, J. Rajadas, M. T. Longaker and G. C. Gurtner, Biomaterials, 2012, 33, 80-90.

18 J. Yang, J. Yeom, B. W. Hwang, A. S. Hoffman and S. K. Hahn, Prog. Polym. Sci., 2014, 39, 1973-1986.

19 M. M. Ayhan, H. Karoui, M. Hardy, A. Rockenbauer, L. Charles, R. Rosas, K. Udachin, P. Tordo, D. Bardelang and O. Ouari, J. Am. Chem. Soc., 2015, 137, 10238-10245.

20 S. J. Barrow, S. Kasera, M. J. Rowland, J. del Barrio and O. A. Scherman, Chem. Rev., 2015, 115, 12320-12406.

21 K. Kim, N. Selvapalam, Y. H. Ko, K. M. Park, D. Kim and J. Kim, Chem. Soc. Rev., 2007, 36, 267-279.

22 M. M. Ayhan, H. Karoui, M. Hardy, A. Rockenbauer, L. Charles, R. Rosas, K. Udachin, P. Tordo, D. Bardelang and O. Ouari, J. Am. Chem. Soc., 2015, 137, 10238-10245.

23 N. Zhao, G. O. Lloyd and O. a. Scherman, Chem. Commun., 2012, 48, 3070.

24 J. Yeom, S. J. Kim, H. Jung, H. Namkoong, J. Yang, B. W. Hwang, K. Oh, K. Kim, Y. C. Sung and S. K. Hahn, Adv. Healthcare Mater., 2015, 4, 237-244.

25 H. Jung, J. S. Park, J. Yeom, N. Selvapalam, K. M. Park, K. Oh, J. Yang, K. H. Park, S. K. Hahn and K. Kim, Biomacromolecules, 2014, 15, 707-714.

26 H. Jung, K. M. Park, J. Yang, E. J. Oh, D. Lee, K. Park, S. H. Ryu, S. K. Hahn and K. Kim, Biomaterials, 2011, 32, 7687-7694.

27 T. D. Henry, B. H. Annex, G. R. McKendall, M. A. Azrin, J. J. Lopez, F. J. Giordano, P. K. Shah, J. T. Willerson, R. L. Benza, D. S. Berman, C. M. Gibson, A. Bajamonde, A. C. Rundle, J. Fine and E. R. McCluskey, Circulation, 2003, 107, 1359-1365.

28 V. Wallenius, M. Hisaoka, K. Helou, G. Levan, N. Mandahl, J. M. Meis-Kindblom, L. G. Kindblom and J. O. Jansson, Am. J. Pathol., 2000, 156, 821-829. 